\title{
Vasa Previa Diagnosis and Management
}

\author{
Andre F. Lijoi, MD, and Joanna Brady, MD
}

Introduction: Vasa previa is an uncommon obstetrical complication that poses a high risk of fetal demise if not recognized before rupture of membranes. It is vital that providers recognize risk factors for vasa previa and diagnose this condition before the onset of labor so that fetal shock or demise is prevented.

Methods: We report a patient with a bilobed placenta and perinatal hemorrhage caused by vasa previa that was not detected with antepartum ultrasound. A review of the literature published between January 1965 and August 2002 was conducted using a MEDLINE-assisted search using the key words "vasa previa," "bilobed placenta," and "succenturiate."

Results: Risk factors for vasa previa have been identified. Advances in ultrasound have led to improved ability to diagnose this condition. Evaluation of patients in high-risk groups with transvaginal color flow Doppler ultrasound should be considered. The accuracy of this technique for diagnosing vasa previa is not known, nor is the true incidence of this condition. Antepartum diagnosis is associated with improved outcomes but does not eliminate morbidity and mortality.

Conclusions: A high index of suspicion for vasa previa at the time of amniotomy is required, because all cases cannot be diagnosed before the onset of labor. ( $\mathrm{J}$ Am Board Fam Pract 2003;16:543-8.)

Vasa previa is an uncommon condition in which fetal blood vessels traverse the lower uterine segment in advance of the presenting part. Neither the umbilical cord nor the placenta supports the vessels. Vasa previa presents with painless vaginal bleeding at the time of spontaneous rupture of membranes or amniotomy (AROM). Fetal shock or demise can occur rapidly. Fetal mortality for cases not recognized before the onset of labor is reported to range between $22.5 \%$ and $100 \%$. ${ }^{1,2}$

A bilobed placenta, divided into 2 approximately equal parts, is uncommon as well. Placentas with 3 or more lobes (multipartite) occur even less frequently. However, those with a small accessory lobe (succenturiate) are more common. Standard obstetrical texts have limited discussions of this topic. Williams Obstetrics includes a brief discussion of the association of vasa previa with placental abnormalities. ${ }^{3}$ Benedetti mentions the association of vasa previa with a succenturiate lobe and anomalies of the umbilical cord but does not discuss the association with a bilobed placenta. ${ }^{4}$ Benirschke and

Submitted, revised, 5 March 2003.

From the Department of Family Practice, York Hospital, York, Pennsylvania. Address correspondence to Andre Lijoi, MD, York Hospital, Thomas Hart Family Practice Center, 1001 S. George Street, York, PA 17405.
Kaufmann do report the potential for vasa previa and note that vascular compression by the presenting part or thrombosis may occasionally cause fetal problems. ${ }^{5}$ Fox does not mention an association with vasa previa. ${ }^{6}$ We report a patient with a bilobed placenta and perinatal hemorrhage caused by vasa previa that was not detected with antepartum ultrasound.

\section{Methods}

A review of the literature published between January 1965 and August 2002 was conducted using a MEDLINE-assisted search with the key words "vasa previa," "bilobed placenta," and "succenturiate."

\section{Case Report}

A 34-year-old woman, gravida 3, para 2, aborta 0 , presented at gestational week 35 , day 2 in active labor. She had a history of a previous precipitous delivery at 36 weeks' gestation. An ultrasound for fetal anatomic survey at 17 to 18 weeks gestation showed a bilobed placenta. Umbilical cord insertion was noted to be normal. Other findings were appropriate.

At 23 weeks' gestation the patient reported a gush of fluid. Work-up did not reveal an explana- 


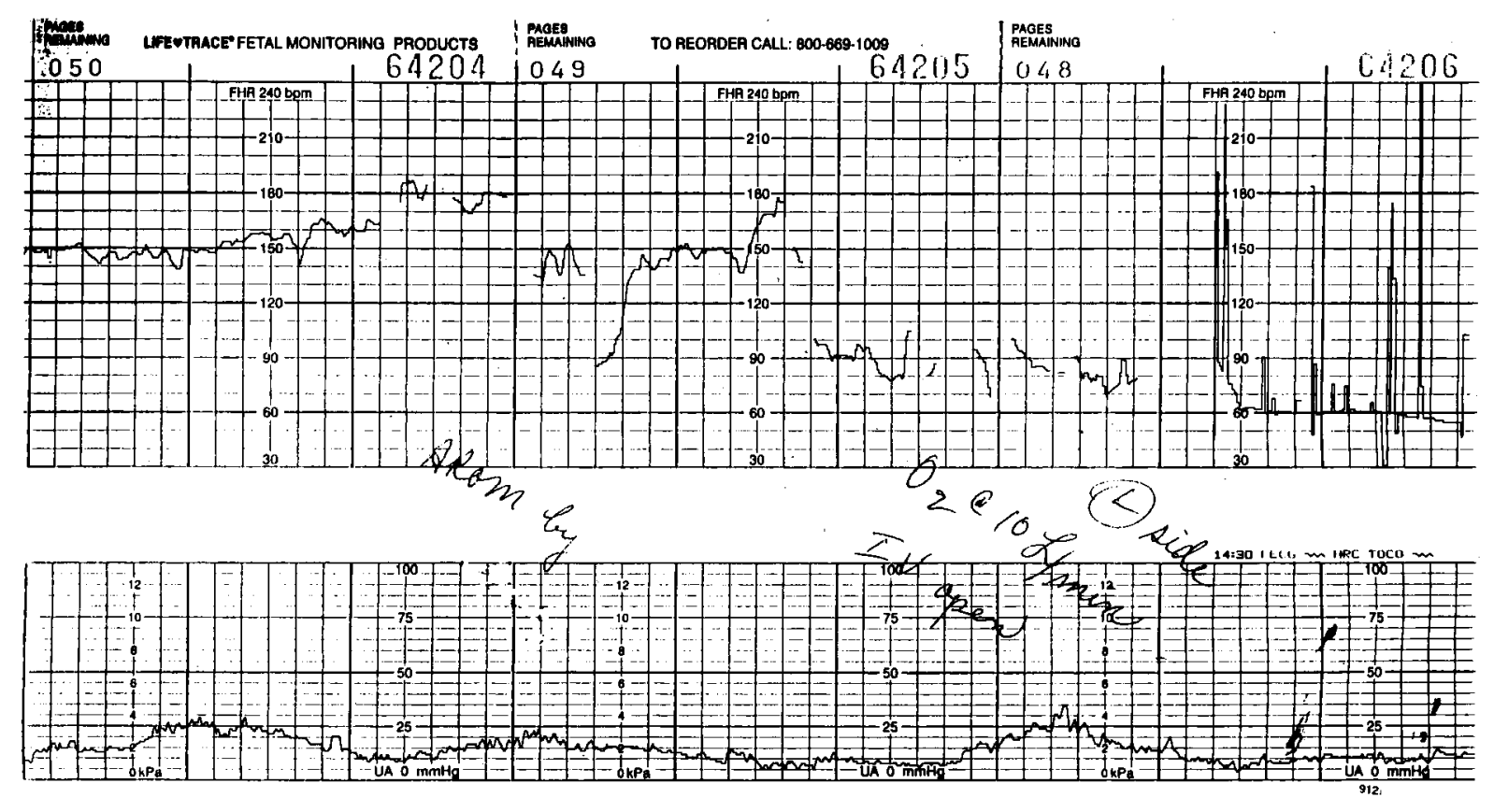

Figure 1. Fetal heart rate tracing from the patient described which shows the rapid development of fetal bradycardia after AROM and subsequent vaginal bleeding.

tion. An ultrasound that included a transvaginal examination showed no abnormality other than the bilobed placenta.

An ultrasound performed at 30 weeks' gestation to assess fetal growth revealed appropriate interval growth and the bilobed placenta. Cervical length, which was assessed because of the history of preterm labor, was normal at $44 \mathrm{~mm}$. Color flow Doppler was not used.

Her labor progressed slowly to 6-cm dilation, at which time a protraction disorder of active labor occurred. Consequently, an AROM was performed, at which time bright red blood with clots was noted. Within 1 minute, the fetal heart rate dropped to 60 to 70 beats/min (Fig. 1). The diagnosis of vasa previa was entertained and the patient was prepared for an emergency cesarean section. Before induction of anesthesia (12 minutes after AROM), the patient delivered vaginally a viable 5-lb, 7-oz boy with Apgar scores of 2/6 at 1 and 5 minutes.

After delivery of the infant, heavy vaginal bleeding occurred. The placenta failed to deliver spontaneously. Manual removal of the placenta was performed under general anesthesia. The mother's hemoglobin level fell from 13.3 to $8.3 \mathrm{~g}$. On inspection of the placenta, 2 separate placental discs with an eccentrically placed cord were noted, along with a torn vessel at the edge of the fetal membranes. The pathologist reported that the cord was marginally inserted on the smaller of the 2 nearly equally sized discs. No large vessels bridging the interplacental membranes were noted. There were vessels traversing the extraplacental membranes.

The child's initial arterial $\mathrm{pH}$ was 7.24 and the hematocrit was 0.28 . The hematocrit dropped to 0.207 . He was treated with iron, did not require transfusion, and was discharged home after a 10 day NICU stay.

\section{Discussion}

Vasa previa is a rare obstetrical catastrophe with a reported incidence ranging from 1 in 1275 to 1 in 8333. ${ }^{1,2}$ The diagnosis is often not made antepartum. Patients usually present with bleeding at the time of spontaneous or artificial rupture of membranes. ${ }^{2}$ However, bleeding can occur before rupture of membranes. ${ }^{7}$ Vasa previa can also present with fetal bradycardia when the velamentous vessels are compressed by the presenting part. ${ }^{8,9}$ Antoine et $\mathrm{al}^{10}$ described a twin pregnancy complicated by vasa previa that presented with a sinusoidal fetal heart rate pattern before the development of bleeding. Occasionally, fetal vessels are palpated at the time of digital examination, and compression of 


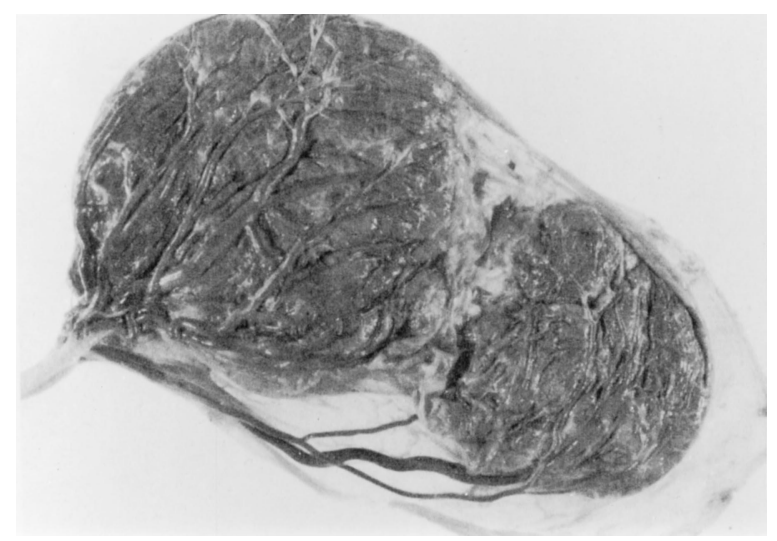

Figure 2. Placenta demonstrating bilobed structure, marginal insertion of umbilical cord, and partial velamentous insertions of cord (fetal vessels traversing membranes to reach smaller placental lobe on right). (Reprinted from Cunningham FG, et al. editors. Williams obstetrics. 21st ed. New York: McGraw-Hill; 2001. p. 828. Copyright () 2001 McGraw-Hill Companies. Reproduced with permission.)

the vessel may cause deceleration of the fetal heart rate. ${ }^{11}$ Fetal death caused by asphyxia and hemorrhagic shock has been described. ${ }^{12,13}$

The reported incidence of bilobed placenta varies widely in the literature, ranging from $0.04 \%$ to $4.2 \%{ }^{6,5}$ In the Collaborative Perinatal Study, Naeye ${ }^{14}$ examined more than 46,000 placentas and found $1.7 \%$ to be bilobed. In two thirds of cases, a velamentous cord insertion was identified (Fig. 2). The cord inserts on the larger placental disk in a third of cases. ${ }^{5}$ The risk of retained placenta is increased. ${ }^{6}$

An amnioscope is a simple tube endoscope that is used for the direct visualization of the amniotic membranes. In their study evaluating postdatism, Browne and Brennan ${ }^{15}$ identified 2 cases of vasa previa using amnioscopy. Barham ${ }^{16}$ reported in 1973 the diagnosis of vasa previa via amnioscopy before amniotomy.

The Kleihauer Betke, Ogita, and Apt tests and hemoglobin electrophoresis can be used to detect the presence of fetal hemoglobin when patients present with vaginal bleeding; however, time often does not allow for this to be completed in an emergent situation. ${ }^{3,4,17,18}$ Magnetic resonance imaging has been used to diagnose vasa previa but is often impractical for diagnosis, especially in the emergent setting. ${ }^{19}$
Many authors have tried to elucidate the role of ultrasound in diagnosing vasa previa. Gianopoulos and colleagues ${ }^{20}$ were the first to describe the diagnosis of vasa previa using ultrasound in a woman with a history of a low-lying placenta and a succenturiate lobe. Nelson and colleagues ${ }^{21}$ were the first to report the use of color flow Doppler to diagnose vasa previa in a woman at 26 weeks' gestation. Clerici and colleagues ${ }^{22}$ also reported the use of color flow Doppler to confirm the presence of vasa previa in a patient that presented with a suspected amniotic band identified by routine ultrasound.

Daly-Jones and colleagues ${ }^{23}$ reported a 34-yearold woman in her second trimester with findings of a low-lying bilobed placenta on ultrasound. Color flow Doppler confirmed the presence of vasa previa. They recommended that women with a lowlying placenta be screened for vasa previa with color flow Doppler.

Between January 1991 and December 1998, Lee et $\mathrm{al}^{24}$ retrospectively reviewed ultrasounds of 93,874 women who had been scanned in their medical center. Eighteen cases of vasa previa were identified. One case associated with velamentous cord insertion was not diagnosed before labor. The earliest diagnosis was made at 15.6 weeks. Three patients had bilobed placentas and 2 had succenturiate lobes. Eight had a placental edge overlying the cervical os. Three of the 18 patients had regression of the vasa previa noted on late third trimester scanning and had uneventful vaginal deliveries. Sensitivity and specificity of ultrasound for diagnosing vasa previa could not be assessed.

Fung and $\mathrm{Lau}^{1}$ reported 3 patients with vasa previa and reviewed 48 cases reported between 1980 and 1997. Thirty-one patients with vasa previa were diagnosed during or after delivery. Of these patients, 20 developed intrapartum bleeding. Eight of the 20 delivered infants with a 5 -minute Apgar score of less than 7. Twelve of the 20 were anemic or required a transfusion, and 2 of 20 died. The fetal mortality in this group was $22.5 \%$.

In 22 patients, vasa previa was diagnosed antenatally. There were no deaths in this group. Of these patients, 16 did not bleed, and none had any of the above complications. Six of the 22 did bleed before delivery, and resulted in 1 infant with an Apgar score less than 7 and anemia. Therefore, their analysis revealed that the fetal loss rate $(P=$ $.033)$, the incidence of 5-minute Apgar scores $<7$ $(P=.033)$, and the incidence of fetal anemia or 
Table 1. Differential Diagnosis of Vasa Previa Suspected on Ultrasound

Chorioamniotic membrane separation

Normal cord loop

Marginal placental vascular sinus

Amniotic band

neonatal blood transfusion $(P=.002)$ were significantly less if the diagnosis was made antenatally.

Catanzarite and colleagues ${ }^{25}$ reported a specificity of $91 \%$ for the sonographic diagnosis of vasa previa. They could not ascertain the sensitivity of ultrasonographic diagnosis of vasa previa because they did not have outcomes data for all pregnancies they scanned.

The authors suggested that screening "transvaginal ultrasound be performed in the late first or early second trimesters with targeted sonography in patients with 'resolving' placenta previa or lowlying bilobed placenta." They recognized that all cases of vasa previa will probably not be identified by this liberal approach. Maternal size, the status of the maternal bladder, and the orientation of vessels as they cross the lower uterine segment may limit visualization of the offending fetal vessels. ${ }^{24,25}$ The differential diagnosis of vasa previa on ultrasound includes chorioamniotic membrane separation, a normal cord loop, a marginal placental vascular sinus, and an amniotic band, all of which can give an appearance similar to vasa previa (Table 1$).^{24}$ Color flow Doppler helps differentiate these conditions.

Oyelese and colleagues ${ }^{26}$ commented that the true incidence of vasa previa is unknown and may be under-reported. Oyelese and colleagues recommended that consideration should be given to screening patients in high-risk groups for vasa previa with transvaginal ultrasound and possibly amnioscopy before rupture of membranes. These patients included those with bilobed and succenturiate placentas, multiple pregnancies, pregnancies resulting from in vitro fertilization, those with low insertion of the cord, and those in which a palpable vessel or a suspected amniotic band is felt on vaginal examination. ${ }^{2}$ Daly-Jones and colleagues ${ }^{23}$ recommended that patients with a low-lying placenta also be screened (Table 2).

Oyelese $^{27}$ also recommended a routine obstetric ultrasound at 20 weeks' gestation and stressed that placental location and cord insertion should be as-

\section{Table 2. Risk Factors for Vasa Previa}

Bilobed and succenturiate placentas

Low-lying placentas

Multiple pregnancies

Pregnancies resulting from IVF

Marginal insertion of the cord

Velamentous insertion of the cord

Palpable vessel or a suspected amniotic band is felt on vaginal exam

certained, given the association of a velamentous or marginal insertion of the cord and vasa previa. Nomiyama et $\mathrm{al}^{28}$ reported that the placental cord insertion could be identified in $99.8 \%$ of $587 \mathrm{pa}-$ tients scanned between 18 and 20 weeks. The sensitivity for identifying velamentous insertion was $100 \%$, the specificity was $99.8 \%$, the positive predictive value was $83 \%$, and the negative predictive value was $100 \%$. Two cases of vasa previa were identified, but only 1 persisted to term. These results contrast with those reported by Pretorius et $\mathrm{al},{ }^{29}$ who were able to identify placental cord insertion in only $67 \%$ of women scanned between 15 and 20 weeks.

\section{Discussion}

Our patient had 3 ultrasound examinations, 2 of which included transvaginal examinations of the cervix. These showed no evidence of vasa previa or an abnormality of the cord. The diagnosis of vasa previa was considered promptly when vaginal bleeding accompanied amniotomy. Fetal bradycardia quickly ensued, attesting to the rapidity with which this hemorrhage can compromise the fetus.

Our patient was unusual because neither an abnormal placental cord insertion nor vasa previa was noted during ultrasound examinations. The application of color flow Doppler may have been helpful in identifying a vessel near or crossing the os.

Obstetrical providers must be aware of the identified risks for vasa previa including a bilobed placenta. Current evidence suggests that antenatal diagnosis of vasa previa is associated with improved outcomes. Evaluation of patients in highrisk groups (Table 2) with transvaginal color flow Doppler should be considered.

When ultrasound is done in the second or third trimester, identification of a velamentous or marginal cord insertion may identify additional patients at risk for vasa previa. If the placental cord insertion 
cannot be identified, color flow Doppler may be useful.

The accuracy of color flow Doppler for diagnosing vasa previa is not known, nor is the true incidence of this condition. A prospective study of the accuracy of ultrasound and color flow Doppler and their effect on the outcome of pregnancies screened is needed to answer these unresolved questions and to determine the extent of benefit of antenatal diagnosis.

Scheduled cesarean section is the preferred method of delivery when vasa previa has been identified antenatally, usually at 37 to 38 weeks, or when fetal lung maturation has been confirmed. ${ }^{2,25}$ In cases in which serial ultrasound examinations show a regression of a velamentous vessel away from the os, some authors would consider an attempt at a vaginal delivery with preparations for immediate operative delivery. ${ }^{25}$ Others would not allow a trial of labor because a vessel remote from the os might tear and result in fetal hemorrhage or demise. $^{24}$

Physicians must be vigilant whenever amniotomy is performed, because all cases of vasa previa cannot be identified antenatally. In this case, a high index of suspicion and immediate delivery followed by aggressive resuscitation were necessary to avoid fetal shock or demise when vaginal bleeding occurred during labor.

We are grateful to Philip M. Bayliss, MD, for his helpful review of the manuscript.

\section{References}

1. Fung TY, Lau TK. Poor perinatal outcome associated with vasa previa: is it preventable? A report of three cases and review of the literature. Ultrasound Obstet Gynecol 1998;12:430-3.

2. Oyelese KO, Turner M, Lees C, Campbell S. Vasa previa: an avoidable obstetric tragedy. Obstet Gynecol Surv 1999;54:138-45.

3. Abnormalities of placentation. In: Cunningham FG, et al., editors. Williams obstetrics. 21st ed. New York: McGraw-Hill; 2001. p. 828-34.

4. Benedetti TJ. Obstetric hemorrhage. In: Gabbe SG, Niebyl JR, Simpson JL, editors. Obstetrics: normal and problem pregnancies. 4th ed. New York: Churchill-Livingstone; 2002. p. 503-38.

5. Benirschke K, Kaufmann P. Pathology of the human placenta. 4th ed. New York: Springer-Verlag; 2000. p. 353-9, 401-4.

6. Fox H. Pathology of the placenta. 2nd ed. Philadelphia: WB Saunders; 1997. p. 64-5.
7. Carp HJ, Mashiach S, Serr DM. Vasa previa: a major complication and its management. Obstet Gynecol 1979;53:273-5.

8. Dougall A, Baird CH. Vasa previa - report of three cases and review of the literature. Br J Obstet Gynaecol 1987;94:712-5.

9. Naftolin F, Mishell DR Jr. Vasa previa: report of 3 cases. Obstet Gynecol 1965;26:561-5.

10. Antoine C, Young BK, Silverman F, Greco MA, Alvarez SP. Sinusoidal fetal heart rate pattern with vasa previa in twin pregnancy. J Reprod Med 1982; 27:295-300.

11. Curl CW, Johnson WL. Vasa previa, antepartum diagnosis. Obstet Gynecol 1968;31:328-30.

12. Cordero DR, Helfgott AW, Landy HJ, Reik RF, Medina C, O'Sullivan MJ. A non-hemorrhagic manifestation of vasa previa: a clinicopathologic case report. Obstet Gynecol 1993;82(4 Pt 2 suppl):698700.

13. Schellpfeffer MA. Improved neonatal outcome of vasa previa with aggressive intrapartum management. A report of two cases. J Reprod Med 1995;40: 327-32.

14. Naeye RL. Disorders of the placenta, fetus and neonate: diagnosis and clinical significance. St. Louis: Mosby-Year Book; 1991. p. 92-158.

15. Browne AD, Brennan RK. The application, value, and limitations of amnioscopy. J Obstet Gynaecol Br Commonw 1968;75:616-21.

16. Barham KA. Amnioscopy amniotomy: a look at surgical induction of labor. Am J Obstet Gynecol 1973; 117:35-8.

17. Apt L. Melena neonatorum. J Pediatr 1955;47:1-5.

18. VanDrie DM, Kammeraad LA. Vasa previa: case report, review and presentation of a new diagnostic method. J Reprod Med 1981;26:577-80.

19. Nimmo MJ, Kinsella D, Andrews HS. MRI in pregnancy; the diagnosis of vasa previa by magnetic resonance imaging. Bristol Med Chir J 1988;103:12.

20. Gianopoulos J, Carver T, Tomich PG, Karlman R, Gadwood K. Diagnosis of vasa previa with ultrasonography. Obstet Gynecol 1987;69:488-91.

21. Nelson LH, Melone PJ, King M. Diagnosis of vasa previa with transvaginal and color flow Doppler ultrasound. Obstet Gynecol 1990;76:506-9.

22. Clerici G, Burnelli L, Lauro V, Pilu GL, Di Renzo GC. Prenatal diagnosis of vasa previa presenting as amniotic band. 'A not so innocent amniotic band.' Ultrasound Obstet Gynecol 1996;7:61-3.

23. Daly-Jones E, Hollingsworth J, Sepulveda W. Vasa praevia: second trimester diagnosis using color flow imaging. Br J Obstet Gynaecol 1996;103:284-6.

24. Lee W, Lee VL, Kirk JS, Sloan CT, Smith RS, Comstock $\mathrm{CH}$. Vasa previa: prenatal diagnosis, natural evolution and clinical outcome. Obstet Gynecol 2000;95:572-6.

25. Catanzarite V, Maida C, Thomas W, Mendoza A, 
Stanco L, Piacquadio KM. Prenatal sonographic diagnosis of vasa previa: ultrasound findings and obstetric outcome in ten cases. Ultrasound Obstet Gynecol 2001;18:109-15.

26. Oyelese KO, Schwarzler P, Coates S, Sanusi FA, Hamid R, Campbell S. A strategy for reducing the mortality rate from vasa previa using transvaginal sonography with color Doppler. Ultrasound Obstet Gynecol 1998;12:434-8.

27. Oyelese Y. Placenta previa and vasa previa: time to leave the Dark Ages. Ultrasound Obstet Gynecol 2001;18:96-9.

28. Nomiyama M, Toyota Y, Kawano H. Antenatal diagnosis of velamentous umbilical cord insertion and vasa previa with color Doppler imaging. Ultrasound Obstet Gynecol 1998;12:426-9.

29. Pretorius DH, Chau C, Poeltler DM, Mendoza A, Catanzarite VA, Hollenbach KA. Placental cord insertion visualization with prenatal ultrasonography. J Ultrasound Med 1996;15:585-93. 